\title{
AN OUTBREAK OF NOSOCOMIAL INFECTION WITH ACINETOBACTER BAUMANNII IN THE NEONATAL INTENSIVE CARE UNIT OF THE DEPARTMENT OF PAEDIATRICS, UNIVERSITY CLINICAL CENTRE TUZLA, BOSNIA AND HERZEGOVINA
}

\author{
Izeta SOFTIĆ ${ }^{1}$, Husref TAHIROVIĆ ${ }^{2}$, Fahrija SKOKIĆ ${ }^{1}$, Nijaz TIHIĆ ${ }^{3}$, Vincenzo DI CIOMMO ${ }^{4}$, \\ Cinzia AURITI ${ }^{5}$
}

${ }^{1}$ Department of Pediatrics, University Clinical Centre Tuzla, Tuzla, Bosnia and Herzegovina, ${ }^{2}$ Department of Medical Sciences of the Academy of Sciences and Arts of Bosnia and Herzegovina, Sarajevo, Bosnia and Herzegovina, ${ }^{3}$ Polyclinic for Laboratory Diagnostics, Department for Microbiology, University clinical centre Tuzla, Tuzla, Bosnia and Herzegovina ${ }^{4}$ Epidemiology Unit, Bambino Gesù Children's Hospital, IRCCS, Piazza S. Onofrio 4, Rome, Italy, ${ }^{5}$ Neonatal Intensive Care Unit, Bambino Gesù Children's Hospital IRCCS, Piazza S Onofrio 4, Rome, Italy

Corresponding author: Izeta Softić

Department of Pediatrics

University Clinical Centre Tuzla

75000 Tuzla

Bosnia and Herzegovina

izeta.softic@bih.net.ba

Tel.: + 38735303714

Fax.: + 38735303740

Received: August 4, 2013

Accepted: September 16, 2013

Copyright (C) 2013 by

University Clinical Center Tuzla.

E-mail for permission to publish: paediatricstoday@ukctuzla.ba
Objective - Outbreaks of sepsis caused by multidrug-resistant Acinetobacter baumannii in neonatal intensive care units have been reported, but rarely from our country. We describe such an outbreak in the Department of Paediatrics of the University Clinical Centre Tuzla in 2012 to investigate risk factors, the mode of transmission and to assess control measures. Setting - An 18 bed, level 3 neonatal intensive care unit in a university affiliated teaching hospital. Patients and methods - Seventeen neonates who developed multidrug-resistant Acinetobacter baumannii nosocomial infection were matched to 17 neonates who were admitted to the same unit without infections, during the outbreak period. Cases and controls were compared for possible risk factors (birth weight, gender, intubation, antibiotic use, etc.). Surveillance cultures were collected from health care personnel and the environment. Results - Six out of the 17 neonates $(35.3 \%)$ died. Surveillance cultures were negative. Seventeen isolates from newborns had the same patterns of resistance. Multidrug-resistant Acinetobacter baumannii was brought into the unit by an infected infant who was transferred from the neurosurgery hospital. Risk factors significantly associated with the infection were: incubator care $(\mathrm{OR}$ 6.66; $\mathrm{p}=0.034$ ), exposure to a central venous catheter (OR 13.75; $\mathrm{p}$ $=0.004)$, mechanical ventilation $(\mathrm{OR} 5.25 ; \mathrm{p}=0.031)$ and exposure to a patient with Acinetobacter baumannii infection (OR 38.40; $\mathrm{p}=0.02$ ). Conclusion - Surveillance cultures for all newborns transferred from other hospitals and isolation measures are important to prevent nosocomial infections and outbreak. Negative environmental and health care worker cultures have to be meticulously analyzed. Cohorting of affected newborns and nursing staff, contact isolation, and environmental cleaning are crucial to control the outbreak.

Key words: Acinetobacter baumannii • Outbreak • Neonates • Intensive care unit. 


\section{Introduction}

Nososcomial infections are one of the most important causes of morbidity and mortality in hospitals. Nosocomial outbreaks due to Acinetobacter baumannii have been described by many authors $(1,2,3,4)$ but never from our region. This is the first report.

Multidrug-resistant Acinetobacter baumannii is a rapidly emerging pathogen in the health care setting. It causes infections, which include bacteremia, pneumonia, meningitis, urinary tract infection and wound infection. The organism's ability to survive under a wide range of environmental conditions and the ability to persist for an extended period of time on surfaces makes it a frequent cause of outbreaks and a healthcare associated pathogen. Risk factors associated with these infections include: invasive procedures, such as the use of mechanical ventilation, central venous or urinary catheters, and broad-spectrum antimicrobials (5). Colonised or infected neonates can affect the likelihood of cross-transmission between patients. Nosocomial occurrence of Acinetobacter baumannii is related to hospital stay and duration, favoured by the selection pressure of previously used antibiotics $(6,7)$.

The aim of this study was to describe an outbreak of nosocomial infection caused by Acinetobacter baumannii from June to November 2012 in the Neonatal Intensive Care Unit of the Department of Pediatrics of the University Clinical Centre Tuzla, Bosnia and Herzegovina.

\section{Patients and methods \\ Clinical setting}

The Neonatal Intensive Care Unit (NICU) of the Department of Pediatrics of the University Clinical Centre Tuzla has 18 beds reserved for patients requiring intensive care.

The University Clinical Centre is a teaching hospital with 1373 beds, serving a popu- lation of 510,353 . It is the referral center for all inborn neonates (4500 deliveries annually) and those born at hospitals in the vicinity, serving also for pediatric surgery. The working situation is characterized by an average of 10 nurses, backed up by a neonatologist on a daily basis and intensive care physicians in the NICU, with one on duty 24-hours. The NICU has area of $293 \mathrm{~m}^{2}$, has one common and two isolation rooms with two sinks in common and one in every isolation room. The admittance of mothers and visitors is limited.

Infections that occur during hospitalization but which are not present nor incubating upon hospital admission are defined as nosocomial. Infections that occur in the following special situations is considered nosocomial: infections acquired in the hospital and which become evident after hospital discharge and newborn infections that are the result of passage through the birth canal (8).

\section{Design of the study}

We undertook a prospective, systematic surveillance of infections in the NICU. This was an unmatched case control study from June to November 2012. A case was defined as any infant hospitalized in the NICU who developed Acinetobacter baumannii nosocomial infection $(\mathrm{n}=17)$. Randomly selected neonates hospitalized during the same period with no diagnosis of infection $(n=17)$ were controls. An individual record was filled out with the patients' demographic data, along with possible predisposing factors, including: gender, birth weight (BW), gestational age, Apgar score at five minutes, age, duration of hospital stay, incubator care, use of antibiotics, mechanical ventilation and the use of a central venous catheter.

\section{Microbiological investigation}

All of the samples taken from the patients included in this study were inoculated onto 
blood agar, McConkey agar and incubated at $35^{\circ} \mathrm{C}$. Typical colonies were further examined with standard microbiological methods. Environmental cultures were taken at the same time, including samples from incubators, working areas, sink areas, mechanical ventilators, aspirators, liquid soap, dummies, infusion solutions and key-boards. Cultures from the hands of the staff working in the intensive care unit were also obtained during the same periods. We took more than 100 samples. Final identification of Acinetobacter baumannii was determined by the Vitek Compact system using Vitek ID GN.

The antibiotic susceptibilities of Acinetobacter baumannii isolates were determined by the Kirbi-Bauer disk diffusion method on Mueller-Hinton agar, using the Clinical and Laboratory Standards Institute guidelines (9). In parallel, each isolate was tested for antibiotic susceptibility with the Vitek Compact System, using a Vitek AST card 240 in order to determine the minimum inhibitory concentrations (MICs). Antibiotic susceptibility was determined for the following antibiotics: amikacin, aztreonam, cefepime, ceftazidime, ciprofloxacin, colistin, gentamycin, imipenem, meropenem, levofloxacin, tobramycin, tigecycline, and trimetoprim/ sulphamethoxasole.

\section{Statistical analysis}

The results are expressed as absolute and relative numbers. The odds ratios (OR) and 95\% confidence intervals (CI) for comparison between cases and controls were estimated with univariate analysis. Statistical significance was defined as a p-value of $<0.05$. Statistical analyses were conducted with SPSS ver. 18.0 (SPSS Inc., Chicago, IL, USA).

\section{Results}

\section{Description of the outbreak and case characteristics}

The outbreak began in June 2012 and extended until November 2012 (Fig. 1). The index case was diagnosed in June. It involved an infant aged 5 weeks, coming from the neurosurgery department. The infant was transferred in the NICU after implantation of a ventriculoperitoneal shunt due to congenital hydrocephalus internus. Ventriculitis developed as a complication after shunt implantation. We treated ventriculitis for 5 weeks unsuccessfully with ceftazidime, amikacin, meropenem and tobramycin. The neurosurgeon recommended open drainage and intrathecal therapy with tobramycin. Acinetobacter baumannii was isolated 11 times from the cerebrospinal fluid (CSF) of the index patient during hospitalization in the intensive care unit. The infant had prolonged hospitalization from $02 / 24$ to $06 / 29 / 2012$, but Acinetobacter baumannii was isolated from the liquor on 06/16/2012. During the outbreak, the same strain (the same susceptibility pattern) of Acinetobacter baumannii was isolated from 17 neonates. It was identified from blood culture (1 case), central venous catheter ( 4 cases), bronho-aspirate and tubes (7 cases), liquor ( 2 cases), urinoculture (1 case) and eye swab (2 cases).

Six out of seventeen neonates died. All of them had one of the risk factors, such as: central venous catheter, tubes, mechanical ventilation. Acinetobacter baumanni was isolated in broncho-aspirate from cases 5, 12, 13, 14, 15 , and in umbilical catheter from case 3. All of them developed clinical signs of severe sepsis. Cases 3 and 10 were on initial antibiotic therapy, the others were treated with multiple broad spectrum antibiotics, including carbapenems and tobramycin (Table 2). 


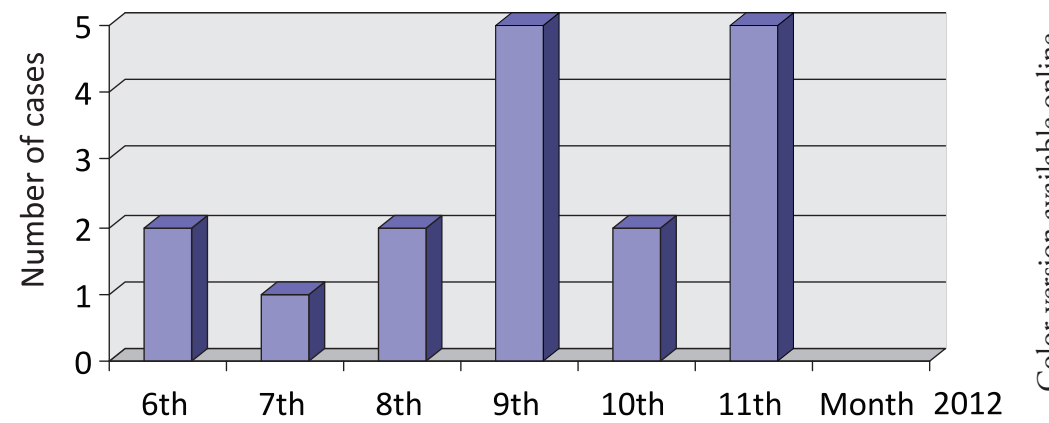

Fig. 1 Distribution of NICU cases, June, July, August, September, October, November.

\section{Table 1 Clinical characteristics of 17 neonates with infections associated with Acinetobacter baumannii}

\begin{tabular}{|c|c|c|c|c|c|c|c|}
\hline Case & $\begin{array}{l}\text { Isolation } \\
\text { date }\end{array}$ & Infection & Diagnosis at admission & $\begin{array}{l}\text { Invasive } \\
\text { procedures }\end{array}$ & Weight (g) & Incubator & Evolution \\
\hline 1 & $06 / 04 / 12$ & Sepsis & Hyprbilirubinaemia & Lumbar puncture & 2350 & + & Survived \\
\hline 2 & $06 / 16 / 12$ & Meningitis & Hydrocephalus & VP shunt & 2200 & - & Survived \\
\hline 3 & $07 / 06 / 12$ & Sepsis & RDS & UVC, Intubation & 2950 & + & Died \\
\hline 4 & $08 / 08 / 12$ & Sepsis & Theratoma colli & Intubation & 2200 & + & Survived \\
\hline 5 & $08 / 29 / 12$ & Sepsis & Meningoencephalitis & UVC, Intubation & 3800 & - & Died \\
\hline 6 & $09 / 10 / 12$ & Sepsis & RDS, Premature & UVC, Intubation & 1100 & + & Survived \\
\hline 7 & $09 / 12 / 12$ & Sepsis & RDS, Premature & UVC & 1650 & + & Survived \\
\hline 8 & $09 / 14 / 12$ & Sepsis & RDS, Premature & UVC & 2010 & + & Survived \\
\hline 9 & $09 / 22 / 12$ & Sepsis & RDS & UVC & 3450 & + & Survived \\
\hline 10 & $09 / 25 / 12$ & Sepsis & RDS & UVC, Intubation & 3000 & + & Survived \\
\hline 11 & $10 / 01 / 12$ & Sepsis & RDS & UVC & 3880 & - & Survived \\
\hline 12 & $10 / 02 / 12$ & Sepsis & RDS, Premature & UVC, Intubation & 2000 & + & Died \\
\hline 13 & $11 / 13 / 12$ & Sepsis & RDS & UVC, Intubation & 3140 & + & Died \\
\hline 14 & $11 / 15 / 12$ & Sepsis & RDS, Premature & UVC, Intubation & 1160 & + & Died \\
\hline 15 & $11 / 21 / 12$ & Sepsis & RDS, Premature & UVC, Intubation & 985 & + & Died \\
\hline 16 & $11 / 28 / 12$ & Sepsis & Ichtiosis & UVC & 2660 & + & Survived \\
\hline 17 & $11 / 29 / 12$ & Sepsis & RDS, Premature & UVC & 1500 & + & Survived \\
\hline
\end{tabular}

All infants received antibiotics. RDS= respiratory distress syndrome; $\mathrm{VP}=$ ventriculo-peritoneal shunt; UVC= umbilical venous catheter.

\section{Epidemiology}

The following risk factors were statistically significant: incubator care, use of central venouse catheter, mechanical ventilation, exposure to the patient with multidrug - resistant (MDR) Acinetobacter baumannii infection. During the period of the outbreak of Acineto- bacter infection we undertook cohort isolation of affected newborns and nursing staff in a separate location with contact precaution measures and environmental cleaning to control the outbreak. Cultures taken from health care workers and environmental sources were negative. The risk factors for Acinetobacter baumannii infection are described in the Table 2. 


\begin{tabular}{|c|c|c|c|c|}
\hline \multirow[t]{2}{*}{ Risk factors } & $\begin{array}{l}\text { Cases } \\
(\mathrm{n}=17)\end{array}$ & $\begin{array}{l}\text { Controls Acinetobacter } \\
\text { baumannii }(\mathrm{n}=17)\end{array}$ & \multirow{2}{*}{$\begin{array}{l}\text { Unadjusted OR } \\
(95 \% \mathrm{CI})\end{array}$} & \multirow{2}{*}{$\mathrm{p}$} \\
\hline & n (\%) & n (\%) & & \\
\hline \multicolumn{5}{|l|}{ Weight at birth (g) } \\
\hline$>1500$ & $13(76.5)$ & $14(82.3)$ & 1.00 (ref.) & \\
\hline$\leq 1500$ & $4(23.5)$ & $3(17.6)$ & $1.44(0.27,7.68)$ & 0.672 \\
\hline \multicolumn{5}{|l|}{ Sex } \\
\hline Female & $8(47.0)$ & $9(53.0)$ & 1.00 (ref.) & \\
\hline Male & $9(53.0)$ & $8(47.0)$ & $1.27(0.33,4.87)$ & 0.732 \\
\hline \multicolumn{5}{|l|}{ Age } \\
\hline$>7$ days & $3(18.0)$ & $0(0)$ & 1.00 & \\
\hline$\leq 7$ days & $14(83.0)$ & $17(100)$ & - & - \\
\hline \multicolumn{5}{|l|}{ Gestation weeks } \\
\hline $26-29$ & $2(11.8)$ & $1(5.6)$ & $2.60(0.21,32.90)$ & 0.461 \\
\hline $30-33$ & $5(29.4)$ & $3(18.0)$ & $2.17(0.42,11.30)$ & 0.359 \\
\hline $34-36$ & $10(58.8)$ & $13(76.5)$ & 1.00 (ref.) & \\
\hline \multicolumn{5}{|l|}{ Hospitalization } \\
\hline Duration $\geq 7$ days & $12(70.6)$ & $14(82.3)$ & $0.51(0.10,2.61)$ & 0.423 \\
\hline \multicolumn{5}{|l|}{ Apgar at 5 minutes } \\
\hline $0-4$ & $2(11.6)$ & $1(5.9)$ & $3.11(0,25,39,54)$ & 0.382 \\
\hline $5-7$ & $6(35.3)$ & $2(11.7)$ & $4.67(0.77,28.41)$ & 0,095 \\
\hline $8-10$ & $9(53.0)$ & $14(82.3)$ & 1.00 (ref.) & \\
\hline Incubator & $15(88.2)$ & $9(52.9)$ & $6.66(1.15,38,46)$ & 0.034 \\
\hline Antibiotic use & $17(100)$ & $17(100)$ & - & - \\
\hline Carbapenem use in NICU & $11(64.7)$ & $0(0)$ & - & - \\
\hline CVC utilization & $15(88.2)$ & $6(35.2)$ & $13.75(2.32,83,3)$ & 0.004 \\
\hline Mechanical ventilation & $9(53.0)$ & $3(17.6)$ & $5.25(1.09,25.21)$ & 0.031 \\
\hline $\begin{array}{l}\text { Exposure to patient with MDR Acinetobacter } \\
\text { baumannii infection }\end{array}$ & $16(94.1)$ & $5(29.4)$ & $38.40(3.95,333.33)$ & 0.002 \\
\hline
\end{tabular}

${ }^{*} \mathrm{p}=0.05$ (statistically significant); $\mathrm{OR}=$ odds ratio; $\mathrm{CI}=$ confidence intervals; $\mathrm{MDR}=$ multidrug resistant; $\mathrm{CVC}=$ central venous catheter.

\section{Microbiological surveillance}

All the Acinetobacter baumannii isolates presented the same resistance profile to the antibiotics: gentamicin, ciprofloxacin, ceftazidime, cefepime, imipenem, meropenem, ampicillin/sulbactam with susceptibility only to tobramycin and tigecycline.

\section{Intervention}

For infants who had been infected with $A c i-$ netobacter baumanni we implemented cohort isolation and a separate medical team took care of these patients. Through environmental cleansing, controlling hand-washing, hygiene and several meetings with infection control staff and microbiologist we managed to control the outbreak.

\section{Discussion}

The bacteria Acinetobacter baumannii has emerged as an important nosocomial pathogen, and outbreaks due to multidrug - re- 
sistant strains have been difficult to control, especially in the setting of intensive care units (10). We have documented an outbreak of MDR Acinetobacter baumannii in a NICU during a six-month period, affecting 17 neonates, with 6 deaths. To define the risk factors for the acquisition of infection, we compared the case-infant group with a randomly selected group of control infants. The following factors were significant: use of incubator care, central venous catheter, mechanical ventilation, and expose to patients with MDR Acinetobacter baumannii infection. Intrahospital transmission of Acinetobacter baumannii has been documented (11). Brito et al. (12) documented that the only independent risk factors for developing a nosocomial MDR Acinetobacter baumanni infection were the use of carbapenem and mechanical ventilation.

Although the outbreak caused by Acinetobacter baumannii was traced to a common source, in agreement with prior studies, the environmental reservoirs indicated in those studies, [suction catheters (13), intubation devices (2), ventilator monitor board, tips of feeding syringes, aerosols and air conditioner (14)] in our study all environmental and hand cultures were negative for MDR Acinetobacter baumannii, but we suspect this was due to some lapses in the procedures for taking material.

Though the dissemination of this pathogen is facilitated by its prolonged survival on inanimate surfaces, high colonization rates among hospitalized patients and frequent contamination of healthcare worker's hands have been observed (2). Another unusual aspect was that the index case was an infant with hydrocephalus internus, transferred from neurosurgery. All documented infections in this outbreak were septicemias in neonates using incubator care, central venous catheter, mechanical ventilation and exposure to the patient with MDR Acinetobacter baumannii infection. We found a mortality $(6 / 17$,
$35.3 \%)$ intermediate between the $22 \%$ and $83 \%$ found, associated with this infection, in neonates from other authors (2), but we have to recognize that, due to the limited sample size, the $95 \% \mathrm{CI}$ is within the range from $14 \%$ to $62 \%$, which is an estimate with very low precision .

Several studies have found that the prior carbapenems use is associated with the subsequent development of MDR Acinetobacter baumannii (15). During the period from June to November 2012, the increased usage of carbapenems was recorded in the infected neonates $(64 \%)$.

The Acinetobacter baumannii isolates were resistant to third and fourth generation cephalosporins, carbapenems and 17 out of 17 were ESBL producers. Multiple mechanisms of acquiring resistance have been described for Acinetobacter baumannii resistant to cephalosporins and carbapenems, including altered penicillin-binding proteins, the presence of metallo beta lactamases, and the loss of porins (16).

\section{Conclusion}

Based on a thorough clinical epidemiological investigation, we realized the need to obtain surveillance cultures for all newborns transferred from other hospitals. Careful isolation measures were important for transferred newborns from other hospital suspected of nosocomial infections. Negative environmental and health care worker cultures have to be meticulously analyzed. Increase in the infection rate due to a particular pathogen is proof of the existence of lapses in infection-control measures, resulting in an increase in crosstransmission between patients. Cohorting of affected newborns and nursing staff, contact isolation, and environmental cleaning were implemented to control the outbreak. The surveillance cultures were collected from all possible sources, but the epidemiological 
investigation was not supplemented by molecular typing, which limited our study.

Authors' contributions: Conception and design: IS, HT; Acquisition, analysis and interpretation of data: IS, CA, VC, NT; Drafting the article IS, HT; Revising it critically for important intellectual content: CA, VC, HT, FS.

Conflict of interest: The authors declares that they have no conflict of interest.

\section{References}

1. Hong KB, Oh HS, Song JS, Lim JH, Kang DK, Son IS, et al. Investigation and control of an outbreak of imipenem-resistant Acinetobacter baumannii Infection in a Pediatric Intensive Care Unit. Pediatr Infect Dis J. 2012; 31(7):685-90.

2. Hosoglu S, Hascuhadar M, Yasar E, Uslu S, Aldudak B. Control of an Acinetobacter baumannii outbreak in a neonatal ICU without suspension of service: a devastating outbreak in Diyarbakir, Turkey. Infection. 2012;40(1):11-8.

3. Visca P, Seifert H, Towner KJ. Acinetobacter infection-an emerging threat to human health. IUBMB life. 2011; 63(12):1048-54.

4. McGrth EJ, Chopra T, Abdel-Haq N, Preney K, Koo W, Asmar BI, et al. An outbreak of carbapenem-resistant Acinetobacter baumannii infection in a neonatal intensive care unit: investigation and control. Infect Control Hosp Epidemiol. 2011;32:34-41.

5. Fournier PE, Richet H. The epidemiology and control of Acinetobacter baumannii in health care facilities. Clin Infect Dis 2006; 42:692-9.

6. Chan PC, Huang LM, Lin HC, Chang LY, Chen ML, Lu CY, et al. Control of an outbreak of pandrug-resistant Acinetobacter baumannii colonization and infection in a neonatal intensive care unit. Infect Control Hosp Epidemiol. 2007;28(4):423-9.

7. Baran G, Erbay A, Bodur H, Ongürü P, Akinci E, Balaban N, et al. Risk factors for nosocomial imipenem-resistant Acinetobacter baumannii infections. Int J Infect Dis. 2000;12(1):16-21.
8. Garner JS, Jarwis WR, Emori TG, Horan TC, Hughes JM. CDC definition for nosocomial infection. Am J Infect Control 1988; 16:128-140.

9. CDC, Healthcare Infection Control Practices Advisory Committee. Management of multidrugresistant organisms in healthcare setting. 2006. Atlanta, GA: US Department of Health and Human Services, CDC Heltcare Infection Control Practices Advisory Committee; 2007.

10. Wadl M, Hechenback K, Holl I, Ziesing S, Pfister $\mathrm{W}$, Beer J, et al. Incrising occurrence of multidrug resistance in Acinetobacter baumannii isolates from four German University Hospitals, 20022006. Infection. 2010; 38:47-51.

11. Manikal VM, Landman D, Saurina G, Oydna E, Lal H, Quale J. Endemic carbapenem-resistant Acinetobacter species in Brooklyn, New York: citywide prevalence, interinstitutional spread and relation to antibiotic usage. Clin Infect Dis 2000; 31:101-6.

12. von Dolinger de Brito D, Oliviera EJ, Abdallah VO, da Costa Darini AL, Filho PP. An outbreak of Acinetobacter baumannii sepsticemia in neonatal intensive care unit of a university hospital in Brazil. Braz J Infect Dis. 2005;9:301-9.

13. Pillay T, Pillay DG, Adhicari M, Pillay A, Sturm AW. An outbreak of neonatal infection with Acinetobacter linked to contaminated suction catheters. J Hosp Infect. 1999; 43:299-304.

14. Mc Donald LC, Walker M, Carson L, Arduino M, Aquero SM, Gomez P, et al. Autbreak of Acinetobacter spp. bloodstream infections in a nursery associated with contaminated aerosol and air conditioners. Pediatr. Infect Dis J. 1998;17:716-22.

15. Nemec A, Krizova L, Maixnerova M, Diancourt L, van der Reiden TJ, Brisse $S$, et al. Emergence of carbapenem-resistence in Acinetobacter baumannii in the Chech Repablic is associated with the spread of multidrug -resistant strains of European clone II. J Antimicrob Chemother 2008;62:484-9.

16. Hsueh PR, Teng LJ, Chen WH, Yu CJ, Ho SW, Luh KT. Pandrug resistant Acinetobacter baumannii causing nosocomial infection in a university hospital, Taiwan. Emerg Infect Dis 2002;8:827-32. 